\title{
Capsaicin analog supplementation is not effective to improve 10-km running time-trial performance in amateur athletes: a randomized, crossover, double-blind and placebo-controlled study
}

\section{Ana Elisa von Ah Morano}

Universidade Estadual Paulista Julio de Mesquita Filho

Camila S. Padilha

Universidade Estadual Paulista Julio de Mesquita Filho

Vinicius Aparecido Matos Soares

Universidade Estadual Paulista Julio de Mesquita Filho

Fabiana Andrade Machado

Universidade Estadual de Maringa

Peter Hofmann

Karl-Franzens-Universitat Graz

Fabrício E Rossi ( $\square$ fabriciorossi@ufpi.edu.br)

https://orcid.org/0000-0002-0594-2529

\section{Fabio Lira}

Universidade Estadual Paulista Julio de Mesquita Filho https://orcid.org/0000-0002-9645-1003

Research article

Keywords: pepper, running races, ergogenic aid

Posted Date: September 16th, 2020

DOI: https://doi.org/10.21203/rs.3.rs-45649/v2

License: (c) (i) This work is licensed under a Creative Commons Attribution 4.0 International License. Read Full License 


\section{Abstract}

Background: To investigate the acute effects of a capsaicin analogue supplement on 10-km time trial performance and physiological responses in amateur athletes.

Methods: Twenty-one participants (age = $29.3 \pm 5.5$ years), completed two randomized, double-blind trials: capsaicin analogue condition [Capsiate $(C A P)=24 \mathrm{mg}$ ] or a placebo condition. The participants consumed two doses of $12 \mathrm{mg}$ of capsaicin or placebo capsule 45 minutes before and immediately at the start of each trial. The time required to complete $10-\mathrm{km}$, lactate concentration, maximum heart rate $\left(\mathrm{HR}_{\text {peak }}\right)$, and rating of perceived exertion (RPE) were recorded.

Results: $10-\mathrm{km}$ time-trial performance (CAP $=45.07 \pm 6.41 \mathrm{~min} v s$. placebo $=45.13 \pm 6.73, P=0.828)$ was not statistically significant different between conditions. No statistically significant differences between conditions were detected for lactate concentration $(P=0.507), \operatorname{HR}_{\text {peak }}(P=0.897)$ and $\operatorname{RPE}(P=0.517)$.

Conclusion: Two doses of $12 \mathrm{mg}$ Capsaicin analogue supplementation did not improve performance and physiological responses in a 10-km running time-trial in amateur athletes.

\section{Background}

Long-distance running is one of the most common individual sports modalities and have become even more popular in the last decade [1]. Long-distance running promotes several systemic and muscle adaptations [2], increases maximal oxygen uptake $\left(\mathrm{VO}_{2 \max }\right)$ [3] and time to exhaustion [4]. The oxidative system demand of long-distance runners is about 3 -fold higher compared with physically active people $[5,6]$ and a relative increase of type lla at expense of type IIb, results in an increased fat metabolism [7]. Moreover, long-distance running provokes a calcium imbalance, which results in inefficiency of muscular contraction [8]. Therefore, different ergogenic and thermogenic compounds were strategically used to optimize performance in middle and long-distances running [9].

Capsiate (CAP) is an analogue of capsaicin, belonging to an 8-methyl-N-vanillyl-trans-6-nonenamide natural phytochemical compound found primarily in red peppers [10] and also in the analogue of a sweet pepper species $(\mathrm{CH}-$ 19 sweet) which has the same molecular structure as capsaicin, except for the substitution of $\mathrm{NH}$ for $\mathrm{O}$ in the alkyl chain that alters the pungency factor [11]. This phytochemical has been used as a nutritional strategy to improve performance in different intensities and exercises distances $[12,13]$.

The dose of capsaicin is not well established in humans. Previous studies from our research group found an improvement in performance applying $12 \mathrm{mg}$ of CAP 45 minutes before different types of exercise and protocols (400 meters; 1.500 meters; 3.000 meters and high-intensity intermittent exercise) in physically active men [12-14]. Additionally, improvements in combined exercise protocols, such as 5-km running plus strength exercise were detected applying $24 \mathrm{mg}$ of CAP in two doses of $12 \mathrm{mg}$ with 45 minutes interval between doses [15]. On the other hand, another study showed improved endurance performance using $150 \mathrm{mg}$ CAP administered 60 minutes before a 30 min aerobic cycle ergometer exercise bout, demonstrating higher fat oxidation under CAP conditions compared to placebo [16]. Peak concentration of CAP is suggested to occur after about 45 minutes after ingestion and bioavailability is approximately 25 minutes, with increased plasma values for up to 105 minutes $[17,18]$.

In skeletal muscle, CAP phosphorylates the transient vanilloid-1 receptor (TRPV1), which provokes the release of available calcium from the sarcoplasmic reticulum, optimizing myosin-muscle actin interaction, improving the processes of force generation and optimizing the efficiency in depleting intramuscular triglycerides. These effects 
preserve g muscle glycogen, and consequently increases time to exhaustion [19]. Moreover, TRPV1 is activated in the putative pain neural circuit [20] increasing, at least in part, fat metabolism due to b-adrenergic stimulation induced by CAP supplementation. Therefore, CAP ingestion may increase fat metabolism by both stimulating lipolysis and inhibiting lipogenesis [21], which could potentiate longer distance performance.

Regarding the potential ergogenic effect of CAP, previous studies from our research group demonstrated that $12 \mathrm{mg}$ of acute CAP supplementation was effective to improve 1.500 meters time-trial running performance with a lower rate of perceived exertion (RPE) [13]. Recently, an investigation about running performance and CAP verified the acute effect of CAP supplementation on short (400 meters) and moderate distance (3.000 meters) running time trial performance in physically active men but did not find differences in heart rate (HR) and RPE [14]. On the other hand, Padilha et al. [22] observed that the acute CAP supplementation did neither increase time to exhaustion during high-intensity continuous

exercise at $90 \%$ of $\mathrm{VO}_{2 \text { peak }}\left(90 \% \mathrm{sVO}_{2 \text { peak }}\right)$ and nor alter physiological responses in runners. Despite several studies investigating the potential effect of CAP on performance primarily in rodents [23], short and middle-distances [13, 21] or high-intensity intermittent exercises in human $[12,24]$ no information is available beyond a certain distance about the effects of the CAP supplementation. Furthermore, previous studies demonstrating the benefits of CAP on performance were conducted in physically active men, therefore, however there is a lack of studies investigating the effect in experienced runners.

Thus, the objective of this study was to verify the acute effect of CAP supplementation on 10-km running time trial performance and physiological responses in amateur athletes. We hypothesize that acute CAP supplementation may be a viable strategy improving long-distance performance $(10-\mathrm{km})$ with lower lactate concentration, $\mathrm{HR}_{\text {peak }}$, and RPE.

\section{Methods}

\section{Experimental design}

This study was a randomized, crossover, double-blind and placebo-controlled study with experimental trials conducted in the morning ( 6 to 9 AM) under similar weather conditions (relative humidity: 60-90\%; wind: 5-29 $\mathrm{km} \cdot \mathrm{h}^{-1}$; temperature: $19-26^{\circ} \mathrm{C}$; altitude: 475 meters) to minimize the chronobiological variance. The participants were instructed about the protocol and were requested to maintain their training and nutritional habits during the study. The participants visited the track for two 10-km running tests under CAP or placebo conditions separated by one week. The trials were performed on a 400 meter official outdoor track, between March to May (in total, it took eight weeks to have all data collected completed).

\section{Participants}

They were advised to abstain from chili peppers or other spicy foods, caffeinated, supplements or ergogenic substances, energy drinks (which contain any kind of stimulant ingredient) or alcoholic beverages as well as strenuous physical exercise within 24 hours prior to testing. Subjects were requested to maintain their regular food intake, and to maintain the same physical exercise regimen 48 hours prior to testing. Additionally, they were instructed to consume breakfast at home as usual, before each experimental trial. Overall energy consumption (kilocalories) and macronutrient intake was calculated from the Brazilian food composition table (TACO) to ensure a similar intake in both experimental trials. The Research Ethics Committee (protocol number 3.654.560/2019) approved the study 
protocol which was conducted according to the 2013 Revision of the Declaration of Helsinki. The subjects signed a consent form and were informed about the purpose of the study and possible risks.

\section{*** INSERT FIGURE 1 ***}

\section{Procedures}

\section{Supplementation protocol}

A researcher who did not participate in the study (double-blinded) randomized the participants by a random sequence generator (www.Random.org) in placebo and CAP condition trial. The second trial was performed in a cross-over manner. In both conditions, subjects ingested two capsules of $12 \mathrm{mg}$ of CAP (24 mg) or placebo according to our previous study that demonstrated benefits of such an application rate [26]. Participants ingested one capsule 45 minutes before the test and the second one immediately before the start of the $10-\mathrm{km}$ running test. This strategy of CAP supplementation was applied to use peak concentration occurring at about 45 minutes after intake and a bioavailability of approximately 25 minutes, to remain the plasma level elevated up to 105 minutes $[17,18]$. The product used for this study contained $50 \%$ extract from capsicum (capsicum annuum L.) from India (Purifarma-Gemini Pharmaceutical Industry Ltda, Anapolis, GO, Brazil) and 50\% of maltodextrin. The correction factor in assay calculation was used by Pharma Nostra (Campinas, Brazil) to guarantee 100\% of capsinoids (Capsiate) in each capsules of 12 mg. The capsules were identical and without flavour. It was delivered to each subject by an independent person who did not participate in the research team in order to secure double-blinding.

\section{0-km time-trial running test}

All participants were experienced in long-distance running and familiar with the track where the tests were performed. All subjects performed the trials 1 and 2 without the presence of opponents or another competitor on the track. Each trial was preceded by a similar self-determined warm-up of $10 \mathrm{~min}$. All participants were encouraged to give their best performance and were cheered by the research team continuously. Participants freely choose their pacing strategy during the run and the split times were recorded every 400 meters. The overall mean velocity for each trial was calculated by dividing the total distance covered by the total time of the test. All subjects had access to mineral water during the run ad libitum.

\section{Blood lactate}

Twenty-five microliters of blood were collected from the volunteer's right ear lobe before, immediately after, and 3, 5 and 7 minutes after each trial. The lactate concentration was determined by electroenzymatic methods using an automated analyser (YSI 2300 STAT ${ }^{\circledR}$, Yellow Springs, Ohio, EUA). Peak lactate concentration was defined for each participant as the highest post-exercise lactate concentration value [27].

\section{Rating of perceived exertion and heart rate}


Rating of perceived exertion was evaluated by the 6-20 points Borg scale and peak of heart rate (Polar ${ }^{\circledR}$ Vantage NV, Electro Oy, Finland) was recorded immediately after the $10-\mathrm{km}$ run was completed.

\section{Incremental test}

To determine aerobic fitness, the participants performed a maximal incremental test on the treadmill (Inbramed ATL ${ }^{\circledR}$,

Porto Alegre - Brazil) until exhaustion. Maximum oxygen uptake $\left(\mathrm{VO}_{2 \mathrm{max}}\right)$ was determined as the mean of the latest 30 seconds from the last stage completed in the incremental test. Gas exchange variables were measured breath-bybreath using a gas analyzer (Model Quark PFT Ergo - Cosmed ${ }^{\circledR}$ - Rome, Italy). Before each test, the gas analyser was calibrated according to the manufacturer's recommendations. The participants performed three minutes of warm-up at $8 \mathrm{~km} \cdot \mathrm{h}^{-1}$. Each stage of the test lasted one minute and the first stage was performed at $9 \mathrm{~km} \cdot \mathrm{h}^{-1}$, with speed increments of $1 \mathrm{~km} \cdot \mathrm{h}^{-1}$ per stage until voluntary exhaustion [28]. The maximal velocity $\left(\mathrm{V}_{\mathrm{max}}\right)$ was assumed as the highest velocity in the stage completed before exhaustion in the incremental exercise test. Mean $V_{\text {max }}$ was compared to the mean velocity of the 10-km running time trial test (Table 2 ).

\section{Body composition and anthropometry}

All the procedures were performed by the same person in an acclimatized room. Body mass was measured on an electronic scale (Filizzola ${ }^{\circledR}$ PL 150, Filizzola Ltda, Brazil). Body height was measured with a wall-mounted stadiometer (Sanny ${ }^{\circledR}$, São Paulo, Brazil). Fat-free mass and fat mass were estimated by bioelectrical impedance following the procedure of the manufacturer (Bia Analyzer $\mathrm{TM}^{\circledR}$, The Nutritional Solutions Corporation, Harrisville, MI, USA).

\section{Statistical analysis}

The sample size of this study was calculated according to previous studies from our research group that verified the improvement of running time in CAP conditions $[13,14]$. An effect size of 0.60 as well as $\beta-1=0.85$ were considered for the power analysis using $G *$ Power software. A sample size of $n=21$ was calculated to sufficient to detect the prescribed effects within this study protocol.

Data were analysed using Statistical Package for the Social Sciences (SPSS ${ }^{\circledR}$ v.24, Inc., Chicago, IL, USA). Data normality was verified using the Shapiro-Wilk test. Data are reported as means and \pm standard deviation (SD). The intraclass correlation coefficient (ICC) test was applied to verify the reliability between trial 1 and 2 (blinded). A paired $t$ test was used to compare both conditions (CAP and placebo) in case of normally distributed data. Statistical significance was set at $P<0.05$.

\section{Results}

Participant's general characteristics are presented in Table 1. There was no difference between trials for total kilocalorie and macronutrient intake 24 hours before exercise for each condition $(P>0.05)$ (Table 2 ).

*** INSERT TABLE 1 AND 2*** 
The first and the second test of the trial were compared in order to identify the influence of learning effects (ICC $=0.98$, $P<0.001)$. No significant differences between the first and the second attempt of the $10-\mathrm{km}$ runs were found $(P>0.05)$ (Table 3). Comparing the effects of CAP versus placebo conditions on 10-km running time did not reveal any significant differences between conditions. Additionally, no statistically significant differences were detected for lactate concentration, $\mathrm{HR}_{\text {peak }}$ and RPE between conditions.

\section{*** INSERT TABLE 3***}

The pacing strategy (mean velocity for each $400 \mathrm{~m}$ lap) for the 10-km running time-trial was the same in both trials for both conditions (placebo and CAP) adopted by the participants, except for the $16^{\text {th }}$ round (where they complete 6400 meters of the trial). At that point, exclusively, the participants running time was faster in CAP when compared to the placebo condition (CAP $=1.49 \pm 0.001 \mathrm{~min} v s$. placebo $=1.52 \pm 0.004 \mathrm{~min}, P<0.05$ ) but overall performance was not significantly affected (Figure 2 ).

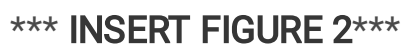

\section{Discussion}

According to our knowledge, this is the first study to investigate the acute effect of CAP supplementation on 10-km time trial performance and physiological responses in amateur athletes. Our main findings regarding CAP supplementation effects on performance are in contrast to previous studies from our research group investigating these effects for shorter distances. The study conducted by de Freitas et al. [14] demonstrated that CAP supplementation was efficient to improve 1500 m time-trial performance with lower RPE but no changes of lactate concentrations between conditions in physically active adults. In addition, acute effects of CAP supplementation were verified to improve performance of short (400 meters) and moderate distance (3.000 meters) running time-trials without changes of $\mathrm{HR}_{\text {peak }}$ and RPE in physically active men.

Therefore, we need to adapt our initial hypothesis that CAP supplementation has an ergogenic effect improving performance and physiological responses in athletes performing a $10 \mathrm{~km}$ time-trial.

Interestingly, in these previous studies, CAP improved 1500-m running time-trial performance by $1.35 \%$, decreased running time by $1.04 \%$ in a 400 -m running time-trial and $2.32 \%$ in a 3.000 meters running time-trial in physically active men, respectively [14]. From these data we might expect an even larger effect on 10-km running time-trial performance as the increase in performance was suggested to be dependent on distance. However, in the current study, there were no significant effects of acute CAP on performance and physiological responses during a 10-km running time-trial in amateur athletes. Furthermore, pacing the $10-\mathrm{km}$ was the same in both conditions, except for a single and small difference at 6.400 meters, where the subjects running time was faster in the CAP condition. Indeed, these findings suggest that the duration of effort (short, middle and longer distance), as well as the training status of subjects, may be important factors with regard to the potential ergogenic benefits of CAP supplementation on exercise performance. 
Our previous studies [12-14] demonstrated benefits of acute CAP application in physically active subjects but not in the current study with amateur athletes.

Contrary, several animal studies have shown, that acute capsaicin supplementation was efficient to improve endurance capacity (exercises protocols between 30-60 minutes of moderate aerobic exercise) [23]. The physiological mechanisms explaining these effects have been related in part to an increase in hepatic glycogen content, being considered an important mechanism for energy homeostasis [23]. Additionally, the activation of TRPV1 due to the capsaicin supplementation and improvements of energy metabolism by increasing lipolysis and saving and maintaining muscle glycogen reserves were suggested [23]. Kazuya et al. [29] demonstrated that 100 mg of CAP improved the control exerted from adenosine diphosphate (ADP) on mitochondrial respiration, facilitating the potent CAP-induced activation of mitochondrial decoupling processes, which leads to the dissipation of the proton gradient generated throughout the respiratory chain. Additionally, oxidative ATP contribution in electrostimulation-induced muscle was enhanced and ATP cost of twitch force generation was reduced. Acute CAP supplementation also enhanced the twitch force-generating capacity [29]. It may be therefore suggested, that acute CAP supplementation can contribute to performance postponing fatigue in resistance exercises [30]. Although these studies showed evidence for CAP supplementation effects on endurance performance, we did not find any improvements in our 10-km running timetrial.

All subjects were encouraged to run as fast as possible giving their best for an excellent performance, however, it was not an official race and therefore motivation influences cannot be ruled out. As an additional limitation we need to mention, that several related markers such as potential hydrogenic $(\mathrm{pH})$, inorganic phosphate, glycogen depletion, calcium release or catecholamine response have not been measured which need to be included in subsequent studies to increase the understanding about CAP supplementation and endurance running performance.

\section{Conclusions}

In conclusion, our study failed to show an ergogenic effect of acute CAP ingestion on time to exhaustion, blood lactate concentration, heart rate response or perceived exertion during a 10-km running time-trial in amateur athletes at the given application rate. Being the first to investigate the effects of acute CAP supplementation on 10-km running in humans, highlights the need to investigate variable doses of CAP supplementation on the activation of the TRPV1 receptor and the subsequent effects on human metabolism in endurance exercise in more detail.

\section{List Of Abbreviations}

ADP Adenosine diphosphate

ATP Adenosine triphosphate

CAP Capsaicin analog

HR Heart rate

RPE Rate of perceived exertion

TRPV1 Transient vanilloid-1 receptor

Vmax Maximum velocity

$\mathrm{VO}_{2 \max }$ Maximum oxygen uptake 


\section{Declarations}

\section{Acknowledgements}

We would like to thank all of subjects who participated in this study as well as the dietician supervision from João Neves de Oliveira.

\section{Authors' contributions}

Study design and organization of the manuscript were performed by AEVAM, VAMS, CSP, FER and FSL. Data analysis, statistical analysis, and the first draft of the manuscript were performed by AEVAM, CSP, FER. The manuscript review was performed by AEVAM, CSP, FER, FAM, PH and FSL. The final approval for publication was performed by FSL.

\section{Funding}

This study was financed in part by the Coordenação de Aperfeiçoamento de Pessoal de Nível Superior-Brasil (CAPES) -Finance Code 001. The funding agency CAPES had no role in the experimental design used, data collection, and interpretation of results or concluding statements

\section{Availability of data and materials}

Data and publication materials can be provided upon request. Please contact corresponding author for this information.

\section{Ethics approval and consent to participate}

Ethics approval for this study was approved by Ethics Research Group of the Federal University of Piauí, Teresina-PI, Brazil (Protocol number: 3.654.560/2019). All participants provided written consent before participating in the study.

\section{Consent for publication}

Not applicable.

\section{Competing interests}

The authors declare no competing interests.

\section{References}

1. Hagan JC. Marathon Running: An Unhealthy Addiction! Mo Med. 2018;115(2):96-7.

2. Li F, Wang R, Newton RU, Sutton D, Shi Y, Ding H. Effects of complex training versus heavy resistance training on neuromuscular adaptation, running economy and 5-km performance in well-trained distance runners. PeerJ. 2019;7:e6787. 
3. Paavolainen L, Nummela A, Rusko H. Muscle power factors and VO2max as determinants of horizontal and uphill running performance. Scand J Med Sci Sports. 2000;10(5):286-91.

4. Hausken K. Evolutions in the physiology of skiing, skating and running in the Olympics. J Sports Med Phys Fitness. 2019;59(7):1175-94.

5. Skovgaard C, Christensen PM, Larsen S, Andersen TR, Thomassen M, Bangsbo J. Concurrent speed endurance and resistance training improves performance, running economy, and muscle NHE1 in moderately trained runners. J Appl Physiol (1985). 2014;117(10):1097-109.

6. Vuorimaa T, Häkkinen K, Vähäsöyrinki P, Rusko H. Comparison of three maximal anaerobic running test protocols in marathon runners, middle-distance runners and sprinters. Int J Sports Med. 1996;17 Suppl 2:S109-13.

7. Giovanelli N, Taboga P, Rejc E, Simunic B, Antonutto G, Lazzer S. Effects of an Uphill Marathon on Running Mechanics and Lower-Limb Muscle Fatigue. Int J Sports Physiol Perform. 2016;11(4):522-9.

8. Pearcey GE, Bradbury-Squires DJ, Kawamoto JE, Drinkwater EJ, Behm DG, Button DC. Foam rolling for delayedonset muscle soreness and recovery of dynamic performance measures. J Athl Train. 2015;50(1):5-13.

9. Schubert MM, Astorino TA. A systematic review of the efficacy of ergogenic aids for improving running performance. J Strength Cond Res. 2013;27(6):1699-707.

10. Ludy MJ, Moore GE, Mattes RD. The effects of capsaicin and capsiate on energy balance: critical review and metaanalyses of studies in humans. Chem Senses. 2012;37(2):103-21.

11. Ohnuki K, Haramizu S, Oki K, Watanabe T, Yazawa S, Fushiki T. Administration of capsiate, a non-pungent capsaicin analog, promotes energy metabolism and suppresses body fat accumulation in mice. Biosci Biotechnol Biochem. 2001;65(12):2735-40.

12. de Freitas MC, Billaut F, Panissa VLG, Rossi FE, Figueiredo C, Caperuto EC, et al. Capsaicin supplementation increases time to exhaustion in high-intensity intermittent exercise without modifying metabolic responses in physically active men. Eur J Appl Physiol. 2019.

13. de Freitas MC, Cholewa JM, Gobbo LA, de Oliveira JVNS, Lira FS, Rossi FE. Acute Capsaicin Supplementation Improves 1,500-m Running Time-Trial Performance and Rate of Perceived Exertion in Physically Active Adults. J Strength Cond Res. 2018;32(2):572-7.

14. Costa LA, de Freitas MC, Cholewa H, Nakamura FY, Panissa VLG, Mora e Silva VEL, et al. Acute capsaicin analog supplementation improves 400 and $3000 \mathrm{M}$ running time-trial performance. International Journal of Exercise Science. 2020;13(2):10.

15. de Freitas MC, Cholewa JM, Panissa VLG, Toloi GG, Netto HC, Zanini de Freitas C, et al. Acute Capsaicin Supplementation Improved Resistance Exercise Performance Performed After a High-Intensity Intermittent Running in Resistance-Trained Men. J Strength Cond Res. 2019.

16. Shin KO, Moritani T. Alterations of autonomic nervous activity and energy metabolism by capsaicin ingestion during aerobic exercise in healthy men. J Nutr Sci Vitaminol (Tokyo). 2007;53(2):124-32.

17. Chaiyasit K, Khovidhunkit W, Wittayalertpanya S. Pharmacokinetic and the effect of capsaicin in Capsicum frutescens on decreasing plasma glucose level. J Med Assoc Thai. 2009;92(1):108-13.

18. Rollyson WD, Stover CA, Brown KC, Perry HE, Stevenson CD, McNees CA, et al. Bioavailability of capsaicin and its implications for drug delivery. J Control Release. 2014;196:96-105.

19. Lotteau S, Ducreux S, Romestaing C, Legrand C, Van Coppenolle F. Characterization of functional TRPV1 channels in the sarcoplasmic reticulum of mouse skeletal muscle. PLoS One. 2013;8(3):e58673.

20. Watanabe T, Kawada T, Kurosawa M, Sato A, Iwai K. Adrenal sympathetic efferent nerve and catecholamine secretion excitation caused by capsaicin in rats. Am J Physiol. 1988;255(1 Pt 1):E23-7. 
21. Yoshioka M, Lim K, Kikuzato S, Kiyonaga A, Tanaka H, Shindo M, et al. Effects of red-pepper diet on the energy metabolism in men. J Nutr Sci Vitaminol (Tokyo). 1995;41(6):647-56.

22. Padilha CS, Billaut F, Figueiredo C, Panissa VLG, Rossi FE, Lira FS. Capsaicin Supplementation during Highintensity Continuous Exercise: A Double-blind Study. Int J Sports Med. 2020.

23. Kim J, Park J, Lim K. Nutrition Supplements to Stimulate Lipolysis: A Review in Relation to Endurance Exercise Capacity. J Nutr Sci Vitaminol (Tokyo). 2016;62(3):141-61.

24. Opheim MN, Rankin JW. Effect of capsaicin supplementation on repeated sprinting performance. J Strength Cond Res. 2012;26(2):319-26.

25. Jones AM, Carter $\mathrm{H}$. The effect of endurance training on parameters of aerobic fitness. Sports Med. 2000;29(6):373-86.

26. Conrado de Freitas M, Cholewa JM, Freire RV, Carmo BA, Bottan J, Bratfich M, et al. Acute Capsaicin Supplementation Improves Resistance Training Performance in Trained Men. J Strength Cond Res. 2018;32(8):2227-32.

27. Peserico C, Mezzaroba P, Silva D, Kravchychyn A, Alves J, Machado F. Blood lactate concentrations following maximal incremental test in male runners with different ages. Revista Brasileira de Educação Física e Esporte. 2018;32(1):12.

28. Alves Pasqua L, Damasceno MV, Bueno S, Zagatto AM, de Araújo GG, Lima-Silva AE, et al. Determinant factors of peak treadmill speed in physically active men. J Sports Med Phys Fitness. 2018;58(3):204-9.

29. Kazuya Y, Tonson A, Pecchi E, Dalmasso C, Vilmen C, Fur YL, et al. A single intake of capsiate improves mechanical performance and bioenergetics efficiency in contracting mouse skeletal muscle. Am J Physiol Endocrinol Metab. 2014;306(10):E1110-9.

30. Hsu YJ, Huang WC, Chiu CC, Liu YL, Chiu WC, Chiu CH, et al. Capsaicin Supplementation Reduces Physical Fatigue and Improves Exercise Performance in Mice. Nutrients. 2016;8(10).

\section{Tables}

Table 1. General characteristics of participants

\begin{tabular}{lc}
\hline Variables & $(\mathrm{N}=21)$ \\
\hline Age (years) & $29.3 \pm 5.4$ \\
Weight $(\mathrm{kg})$ & $74.2 \pm 11.3$ \\
Height $(\mathrm{cm})$ & $176.0 \pm 0.0$ \\
Fat mass (\%) & $12.7 \pm 3.8$ \\
Fat Free Mass $(\mathrm{kg})$ & $64.3 \pm 7.2$ \\
$\mathrm{VO}_{2 \mathrm{max}}\left(\mathrm{ml} \cdot \mathrm{kg}^{-1} \cdot \mathrm{min}^{-1}\right)$ & $62.7 \pm 8.4$ \\
\hline
\end{tabular}

Note: Data are presented in mean and \pm SD. 
Table 2. Dietary intake and macronutrient distribution $24 \mathrm{~h}$ before each trial.

\begin{tabular}{lccc}
\hline- & Placebo & CAP & $P$-value \\
\hline Total intake $(\mathrm{kcal})$ & $1.7 \pm 661$ & $1.8 \pm 476$ & 0.322 \\
Protein $(\mathrm{g})$ & $97.2 \pm 41.7$ & $107.2 \pm 47.7$ & 0.221 \\
Carbohydrate $(\mathrm{g})$ & $213.6 \pm 97.4$ & $206.6 \pm 69.3$ & 0.900 \\
Lipids $(\mathrm{g})$ & $61.0 \pm 28.4$ & $66.9 \pm 26.0$ & 0.112 \\
& & & \\
Total intake $\left(\mathrm{kcal}^{\left.-\mathrm{kg}^{-1}\right)}\right.$ & $24.8 \pm 9.2$ & $21.8 \pm 10.9$ & 0.357 \\
Protein $\left(\mathrm{g} \cdot \mathrm{kcal}^{-1}\right)$ & $1.3 \pm 0.5$ & $1.2 \pm 0.7$ & 0.669 \\
Carbohydrate $\left(\mathrm{g} \cdot \mathrm{kcal}^{-1}\right)$ & $3.0 \pm 1.4$ & $2.4 \pm 1.3$ & 0.219 \\
Lipids $\left(\mathrm{g} \cdot \mathrm{kcal}^{-1}\right)$ & $0.8 \pm 0.3$ & $0.7 \pm 0.4$ & 0.660 \\
\hline
\end{tabular}

Note: Data are show in mean and \pm SD. $P<0.05$ compared to placebo condition.

ble 3 Performance and physiological response in 10-km running test at CAP and placebo conditions $(\mathrm{n}=21)$

\begin{tabular}{|c|c|c|c|c|c|c|}
\hline & $\begin{array}{c}\text { Trial } 1 \\
\text { (Blinded) }\end{array}$ & $\begin{array}{c}\text { Trial } 2 \\
\text { (Blinded) }\end{array}$ & $P$-value & Placebo & CAP & $P$-value \\
\hline Time 10-km (min) & $45.21 \pm 6.44$ & $44.99 \pm 6.70$ & 0.429 & $45.13 \pm 6.73$ & $45.07 \pm 6.41$ & 0.828 \\
\hline Mean velocity $\left(\mathrm{km} \cdot \mathrm{h}^{-1}\right)$ & $13.53 \pm 1.92$ & $13.61 \pm 1.98$ & 0.294 & $13.57 \pm 2.00$ & $13.57 \pm 1.91$ & 0.945 \\
\hline $\mathrm{V}_{\max }$ relative to incremental test (\%) & $76 \pm 4.35$ & $76.45 \pm 4.67$ & 0.332 & $76.21 \pm 4.73$ & $76.24 \pm 4.71$ & 0.943 \\
\hline $\mathrm{HR}_{\text {peak }}(\mathrm{bpm})$ & $181 \pm 12.9$ & $181 \pm 12.1$ & 0.798 & $181 \pm 11.2$ & $180 \pm 13.5$ & 0.942 \\
\hline $\mathrm{RPE}_{\text {peak }}(6-20$ point BORG scale ) & $17 \pm 2.0$ & $17 \pm 2.3$ & 0.550 & $17 \pm 2.3$ & $17 \pm 2.0$ & 0.550 \\
\hline $\begin{array}{l}\text { Peak }\left[\mathrm{La}^{-}\right] \\
\left(\mathrm{mmol}\left[\mathrm{L}^{-1}\right)\right.\end{array}$ & $5.0 \pm 1.6$ & $5.5 \pm 1.9$ & 0.219 & $5.2 \pm 1.8$ & $5.2 \pm 1.8$ & 0.950 \\
\hline
\end{tabular}

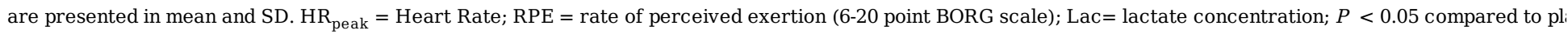

\section{Figures}




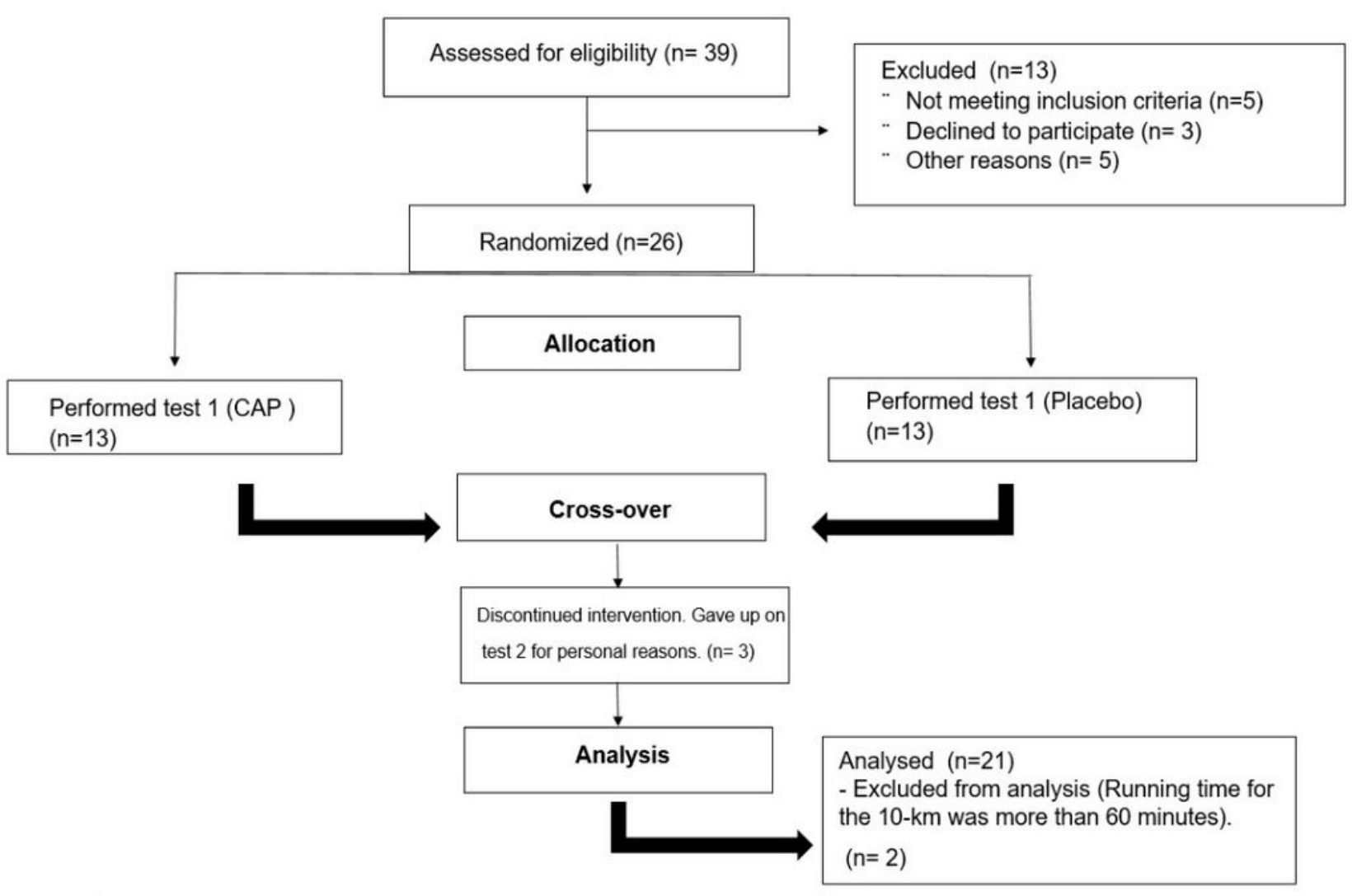

Figure 1

Flow Diagram.

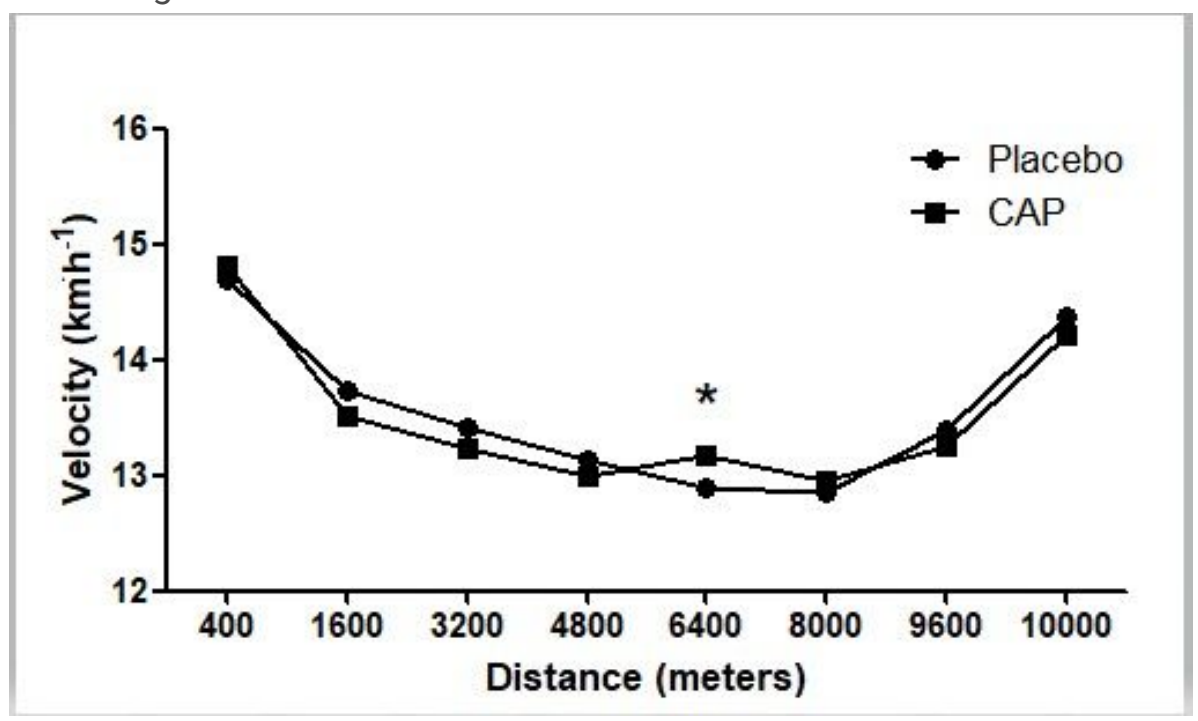

Figure 2

Mean running velocity during a 10-km running time-trial. * $p<0.05$ CAP vs Placebo in same time. 